Article

\title{
Waveguiding Light into Silicon Oxycarbide
}

\author{
Faisal Ahmed Memon ${ }^{1,2, *}$, Francesco Morichetti ${ }^{1}$ and Andrea Melloni ${ }^{1}$ \\ 1 Dipartimento di Elettronica, Informazione e Bioingegneria (DEIB), Politecnico di Milano, via Ponzio 34/5, \\ 20133 Milan, Italy; francesco.morichetti@polimi.it (F.M.); andrea.melloni@polimi.it (A.M.) \\ 2 Department of Telecommunications Engineering, Mehran University of Engineering \& Technology, \\ Jamshoro 76062, Sindh, Pakistan \\ * Correspondence: faisalahmed.memon@polimi.it or faisal.memon@faculty.muet.edu.pk; Tel.: +39-2-2399-8978
}

Academic Editor: Boris Malomed

Received: 1 May 2017; Accepted: 25 May 2017; Published: 30 May 2017

Featured Application: Silicon oxycarbide is demonstrated to be a potential photonic platform for integrated optics applications.

\begin{abstract}
In this work, we demonstrate the fabrication of single mode optical waveguides in silicon oxycarbide $(\mathrm{SiOC})$ with a high refractive index $\mathrm{n}=1.578$ on silica $\left(\mathrm{SiO}_{2}\right)$, exhibiting an index contrast of $\Delta \mathrm{n}=8.2 \%$. Silicon oxycarbide layers were deposited by reactive RF magnetron sputtering of a SiC target in a controlled process of argon and oxygen gases. The optical properties of SiOC film were measured with spectroscopic ellipsometry in the near-infrared range and the acquired refractive indices of the film exhibit anisotropy on the order of $10^{-2}$. The structure of the SiOC films is investigated with atomic force microscopy (AFM) and scanning electron microscopy (SEM). The channel waveguides in $\mathrm{SiOC}$ are buried in $\mathrm{SiO}_{2}(\mathrm{n}=1.444)$ and defined with UV photolithography and reactive ion etching techniques. Propagation losses of about $4 \mathrm{~dB} / \mathrm{cm}$ for both TE and TM polarizations at telecommunication wavelength $1550 \mathrm{~nm}$ are estimated with cut-back technique. Results indicate the potential of silicon oxycarbide for guided wave applications.
\end{abstract}

Keywords: silicon oxycarbide; optical waveguides; optical materials; guided waves; integrated optics

\section{Introduction}

Materials offering refractive index tunability and low absorption are extremely useful for realizing optical integrated devices. In silicon nitride (SiN) compounds, like silicon oxynitride and silicon rich nitride, the refractive index can be tuned over a wide range, potentially from 1.45 to about 2 , yet at the expense of higher losses [1,2]. Silicon oxycarbide (SiOC) is a versatile material that has been used in a variety of applications including Li-ion batteries [3], photoluminescence [4], electroluminescence [5], and low-k interlayer dielectric [6,7]. It has been demonstrated that the change in composition of SiOC compound can lead to a change in the refractive index $\mathrm{n}$ over a wide range from $\sim 1.45$ to $\sim 3.0$ under pre- and post-deposition conditions [8,9]. Structural and chemical properties of SiOC films have been extensively reported in the literature [10-13]. Recently, we have demonstrated the synthesis of SiOC films with reactive RF magnetron sputtering and showed that, under different deposition conditions, the refractive index $\mathrm{n}$ can be varied from 1.41 to 1.85 at wavelength $\lambda=1550 \mathrm{~nm}$, while the extinction coefficient $\mathrm{k}$ is below $10^{-4}$ above $\lambda=1000 \mathrm{~nm}$ [14]. The low extinction coefficient $\mathrm{k}$ of SiOC films indicates the suitability of this material for fabrication of optical waveguides. However, to the best of our knowledge, SiOC has never been exploited as a core material for guided wave applications.

In this paper, we demonstrate the fabrication of silicon oxycarbide optical waveguides with reactive RF magnetron sputtering. Reactive sputtering is a well-established technique, relatively simple and cost effective to set up compared to conventional chemical vapor deposition (CVD). First, 
SiOC films are deposited by sputtering the SiC target in the presence of argon (Ar) and oxygen $\left(\mathrm{O}_{2}\right)$ gases in a controlled process at room temperature. The prepared films are characterized with variable angle spectroscopic ellipsometry over near-infrared wavelength range. The deposited SiOC film exhibits anisotropy on the order of $10^{-2}$ between in-plane and out-of-plane refractive indices as acquired from the ellipsometric data. To quantify the root mean square (RMS) roughness of the deposited $\mathrm{SiOC}$ film on $\mathrm{SiO}_{2} / \mathrm{Si}$ wafer, atomic force microscopy is used. RMS roughness is an important parameter that affects the performance of optical waveguides and contributes to the scattering of light. The sputtered SiOC films are then used as core layer to fabricate channel waveguides using UV photolithography and reactive ion etching (RIE) techniques. The SiOC channel waveguides are buried between two layers of $\mathrm{SiO}_{2}(\mathrm{n}=1.444)$. The optical waveguides with different widths are characterized at an optical communication wavelength $(1550 \mathrm{~nm})$ with different lengths. Propagation losses of about $4 \mathrm{~dB} / \mathrm{cm}$ for both TE and TM polarizations have been achieved indicating the suitability of sputtered SiOC for integrated optics applications.

The paper is organized as follows: Section 2 explains deposition and characterization techniques used to grow and study the properties of SiOC films (Section 2.1), and the fabrication process and the measurement setup for SiOC optical waveguides (Section 2.2). Section 3 discusses results on the characterization of SiOC films (Section 3.1) and optical waveguides (Section 3.2). Finally, conclusions are presented in Section 4.

\section{Experimental Details}

\subsection{Layer Fabrication and Characterizations Techiques}

SiOC films were deposited with reactive RF magnetron sputtering of a silicon carbide ( $\mathrm{SiC}$ ) target in presence of argon (Ar) and oxygen $\left(\mathrm{O}_{2}\right)$ gases at room temperature. The SiOC films are deposited on a 4 inches $\mathrm{Si}(100)$ wafer with $8 \mu \mathrm{m} \mathrm{SiO}{ }_{2}$ buffer layer that isolates $\mathrm{SiOC}$ core from high index $\mathrm{Si}$ substrate. To achieve the desired refractive index, an optimized sputtering recipe with the following parameters is used: $\mathrm{rf}$ power $=350 \mathrm{~W}$, Ar flow $=60 \mathrm{sccm}$, and $\mathrm{O}_{2}$ flow $=2.6 \mathrm{sccm}$. The sputtering was run for around three minutes to obtain a SiOC layer with a thickness of $400 \mathrm{~nm}$. Further details on deposition process, obtained deposition rate of sputtering, and optical parameters of SiOC films are provided in [14].

The surface roughness of the deposited SiOC layer was determined with atomic force microscope (5600LS AFM system, Keysight, CA, USA) at five different places on the four-inch wafer. The SiOC layer was probed in contact mode over an area of 10 by $10 \mu \mathrm{m}^{2}$ at each location. Cross-sectional images of SiOC film and waveguides were obtained with scanning electron microscope (SEM LEO 1525, One Zeiss Drive, NY, USA) to investigate the morphology and verify the profile and thickness. Since SiOC is a dielectric material, a thin layer of platinum $(\approx 2 \mathrm{~nm})$ was deposited on the SiOC films to avoid electrons charging effect. The high-resolution images were captured by accelerating electrons at a voltage of $20 \mathrm{kV}$ and the signal was collected from in-lens detector while keeping a distance of around $6 \mathrm{~mm}$ between the SiOC sample and the electron gun.

The optical properties of the deposited $\mathrm{SiOC}$ film on $\mathrm{SiO}_{2} / \mathrm{Si}$ wafer are measured with variable angle spectroscopic ellipsometer (VASE J.A. Woollam Inc., Lincoln, NE, USA). The ellipsometric data were acquired at multiple incidence angles $\left(65^{\circ}, 70^{\circ}\right.$, and $\left.75^{\circ}\right)$ in the spectral range from 1200 to $1600 \mathrm{~nm}$ with a step size of $10 \mathrm{~nm}$ between two wavelengths. The ellipsometry measures the change in polarization state of the light reflected from the surface of the film/substrate under investigation. The measured quantities are psi $(\psi)$ and del $(\Delta)$ which are angles defining the ratio of Fresnel reflection coefficients $R_{p}$ and $R_{s}$ for parallel ( $p$ ) and normal (s) polarized light as [15],

$$
\frac{R_{p}}{R_{s}}=\tan \psi \exp (i \Delta)
$$


Ellipsomteric measurements were performed taking in to account the possible depolarization originated by thickness non-uniformity and surface roughness. Since ellipsometry is an indirect technique, an optical model is necessary to obtain the optical constants (n, k) of film/substrate. A four-layer film/substrate stack including surface-roughness, $\mathrm{SiOC}$ film, $\mathrm{SiO}_{2}$ layer, and $\mathrm{Si}$ substrate as shown in Figure 1a was used as a model to extract the optical constants $n$ and $k$ of the SiOC film. The surface roughness layer is based on Bruggeman EMA [16,17] that is AFM equivalent and provides peak-to-valley roughness. The SiOC film index was represented by Cauchy dispersion model,

$$
\mathrm{n}(\lambda)=A+\frac{\mathrm{B}}{\lambda^{2}}(\lambda \text { in } \mu \mathrm{m})
$$

where A and B constants are the fit parameters in modelling the measured ellipsometric $\psi$ and $\Delta$ data. The SiOC film was modeled as anisotropic layer by introducing uniaxial anisotropy in the Cauchy model. The optical axis was considered perpendicular to the surface as illustrated in Figure $1 \mathrm{~b}$. From the frame of reference of ellipsometry, ordinary axis $n_{o}$ corresponds to transverse electric (TE) polarization and extra-ordinary axis $n_{e}$ to transverse magnetic (TM) polarization as shown in Figure $1 b$. The data was fitted by the commercial WVASE32 software [17] using the optical model given in Figure 1a. The mean squared error (MSE) is the basis for validating an optical model and determining the quality of the match between the experimental and calculated data. WVASE32 software [17] uses a maximum likelihood estimator with MSE based on the Lavenberg-Marquardt regression algorithm.
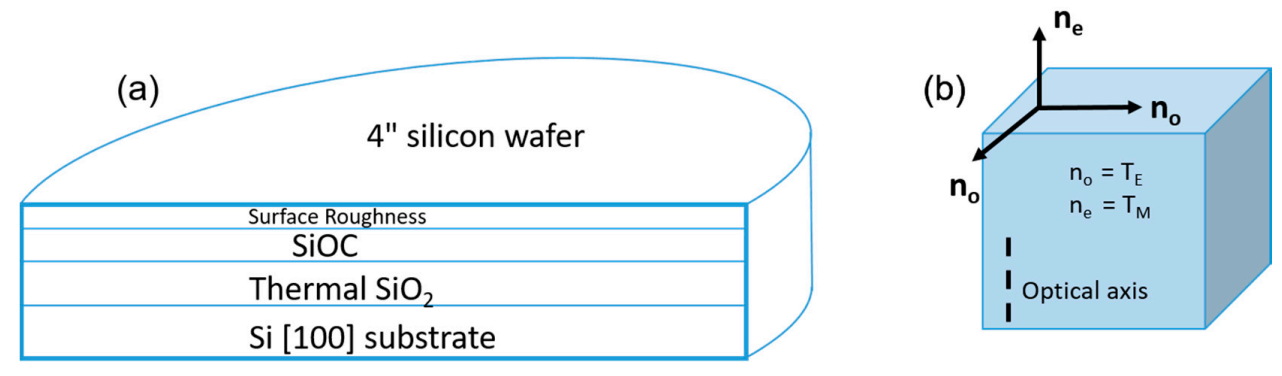

Figure 1. (a) Four-layer optical model employed to retrieve optical constants of SiOC film from ellipsometric data-not to scale, (b) schematic of SiOC anisotropic layer with optical axis perpendicular to surface and ordinary $\mathrm{n}_{\mathrm{o}}$ and extraordinary $\mathrm{n}_{\mathrm{e}}$ refractive indices.

\subsection{Waveguide Fabrication and Characterizations Techniques}

Before fabrication, the geometry of the SiOC waveguides was designed to obtain a single mode operation by using a commercial electromagnetic tool based on Finite Element Method (FEM). The optical waveguides were then fabricated with UV photolithography and reactive ion etching (RIE) processes as follows. To define waveguide patterns, a photoresist AZ $5214 \mathrm{E}$ was spin coated on the SiOC layer and soft baked on a hot plate; then the photoresist was exposed in hard contact mode with photo mask using I-line $365 \mathrm{~nm}$ mercury arc lamp emitting a power of $1000 \mathrm{~W}$. The photo mask includes a set of waveguides with different widths. A controlled RIE process of $\mathrm{CHF}_{3}$ and $\mathrm{O}_{2}$ gases mixture was used to etch channel waveguides in SiOC layer with an etch rate of $20 \mathrm{~nm} / \mathrm{min}$. The realized channel waveguides were covered with $8 \mu \mathrm{m}$ thick $\mathrm{SiO}_{2}(\mathrm{n}=1.444)$ deposited by a plasma enhanced chemical vapor deposition (PECVD) process (STS CVD tool) to yield symmetric optical modes.

The optical waveguides were characterized on the optical bench through butt-coupling with small-core fibers as shown in Figure 2. The schematic diagram of the waveguide measurement setup is given in Figure 2a. The light signal was coupled from a laser source operating around telecommunication wavelength $\lambda=1550 \mathrm{~nm}$. The SiOC waveguides were aligned with small core fibers having mode field diameter MFD $\approx 3.6 \mu \mathrm{m}$ using index matching oil to reduce the gap between the fiber and chip facet. The light at the output facet of SiOC chip was collected and read with optical power meter. The polarization state (TE and TM) of the light wave at the input facet of the waveguide 
was selected with a fiber polarization controller. The real optical waveguide measurement setup is shown in Figure $2 b$ where a SiOC chip is aligned with small core fibers.

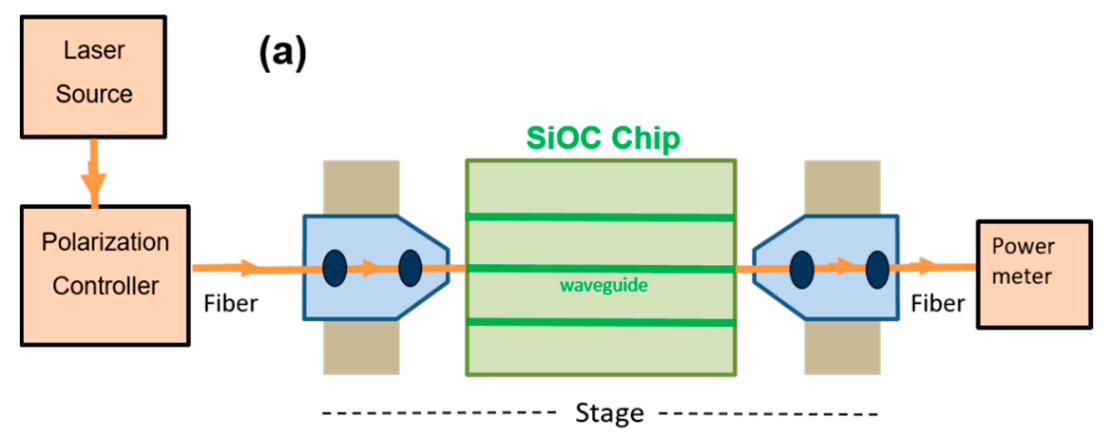

(b)

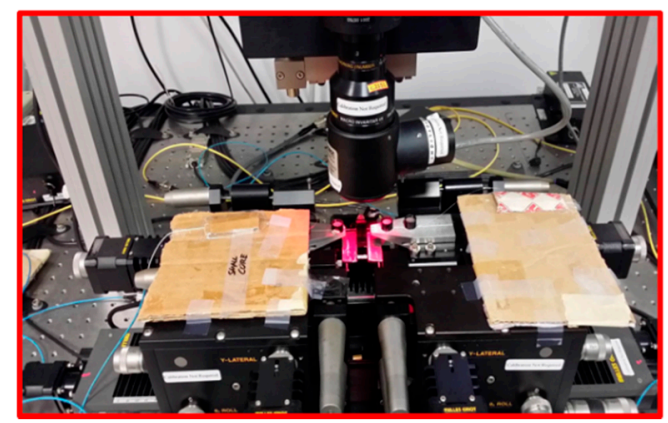

Figure 2. (a) Schematic of optical bench setup used for SiOC waveguides characterizations, (b) the measurement setup showing alignment of SiOC chip with small core optical fibers.

\section{Results and Discussion}

\subsection{SiOC Layer Properties}

SEM cross-section image of the $\mathrm{SiOC}$ film deposited on $\mathrm{SiO}_{2} / \mathrm{Si}$ wafer under the optimized sputter process is displayed in Figure 3. The thickness of the SiOC layer is determined to be $400 \mathrm{~nm}$. The deposited SiOC film shows dense columnar structure with pillars perpendicular to the plane of film. The columnar structure of the film provides a basis for anisotropy investigation and is taken into consideration during the optical characterization of SiOC film. The columnar structure is a typical feature in sputtered SiOC films that were deposited under different conditions, however the films were amorphous to XRD [14].

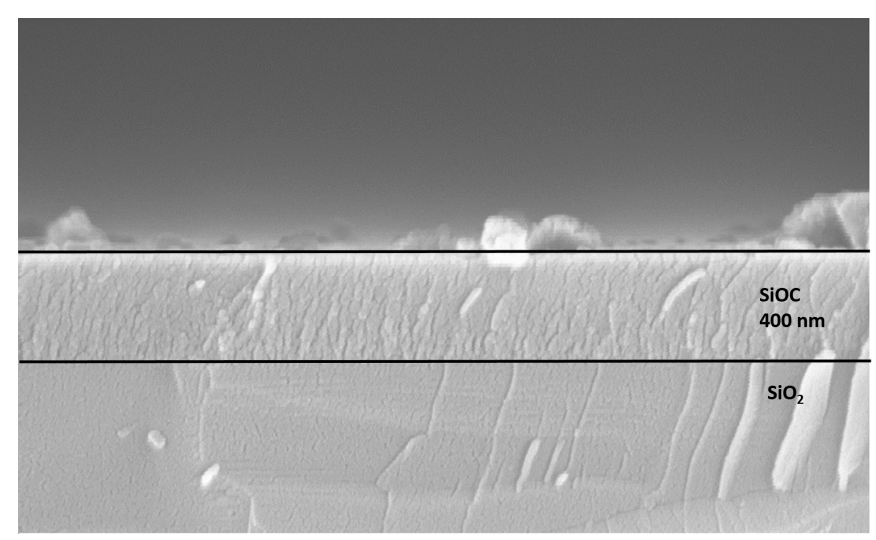

Figure 3. SEM cross-section image of $\mathrm{SiOC}$ deposited layer on $\mathrm{SiO}_{2} / \mathrm{Si}$ wafer. 
Figure 4a shows 3D topographical image of the SiOC film scanned with AFM in contact mode over an area of $100 \mu \mathrm{m}^{2}$. The scans were performed at five different locations on the four-inch wafer and the rms roughness is estimated to be $0.88 \mathrm{~nm}$, sufficiently smooth for the realization of optical waveguides. In Figure 4b, 1D measured profile of the AFM image is given showing peak-to-valley roughness that is in-line with surface roughness estimated with ellipsometry.

(a)

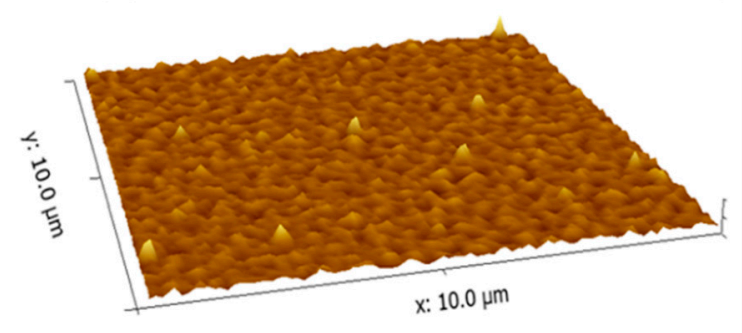

(b)

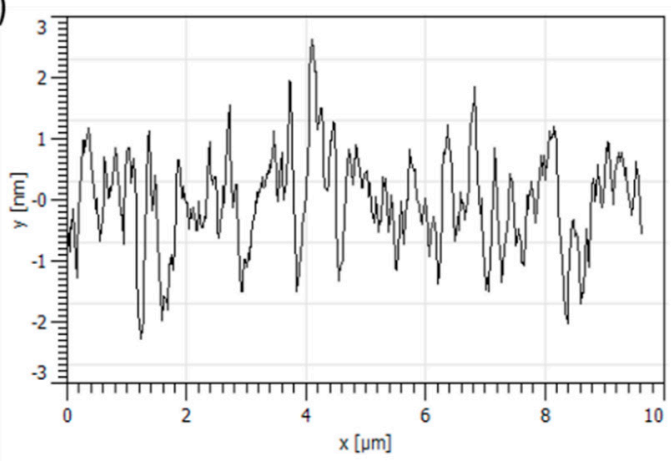

Figure 4. (a) 3D AFM topography scan of SiOC film surface (color scale from $-5 \mathrm{~nm}$ to $+5 \mathrm{~nm}$ ); (b) 1D measured profile of AFM image: a peak-to-valley roughness of about $2 \mathrm{~nm}$ is observed that agrees well with surface roughness estimated with spectroscopic ellipsometry.

The optical properties of the deposited SiOC film were characterized with variable angle spectroscopic ellipsometry in the infrared wavelength region from $1200 \mathrm{~nm}$ to $1600 \mathrm{~nm}$. The measurement angles were $65^{\circ}, 70^{\circ}$, and $75^{\circ}$ which are around the Brewster's angle of Si substrate. Prior to SiOC film, the $\mathrm{SiO}_{2} / \mathrm{Si}$ wafer was characterized with ellipsometry to obtain the thickness and accurate optical constants of $\mathrm{SiO}_{2}$. The refractive index of $\mathrm{SiO}_{2}$ was determined as 1.444 at $\lambda=1550 \mathrm{~nm}$. The tabulated optical constants of crystalline Si substrate were used from the WVASE32 database [17]. Since ellipsometry is sensitive to film roughness conditions, surface roughness (SR) layer [16] was added to simulate roughness on SiOC film which improved fit and MSE. The SR layer that is the ellipsometry equivalent of AFM is estimated around $2 \mathrm{~nm}$ and agrees well with the AFM data. The ellispometric $\psi$ and $\Delta$ data acquired at different angles and their respective model curves are given in Figure 5.

The four-layer anisotropic optical model discussed in Section 2 was used to fit the experimental data (green curves) and the calculated model data (red curves) matches well over the entire spectral range. Material anisotropy is expected from the columnar structure present in the sputtered SiOC film as shown in SEM image (see Figure 3). Our measurements indicate that the out-of-plane refractive index $\left(n_{e}\right)$ is higher than the in-plane index $\left(n_{0}\right)$ by 0.02438 . These results agree with the studies reporting anisotropy in different material thin films with columnar structure where the in-plane refractive index is smaller than out-of-plane refractive index [18-20]. Therefore, the origin of the anisotropy is attributed to the columnar structure oriented normal to the plane of SiOC film. The anisotropy exhibited by SiOC film is on the order of $10^{-2}$, the optical waveguides are sensitive to even this level of anisotropy because the two polarizations (TE and TM) will see a different index of refraction and propagate with different effective indices. 

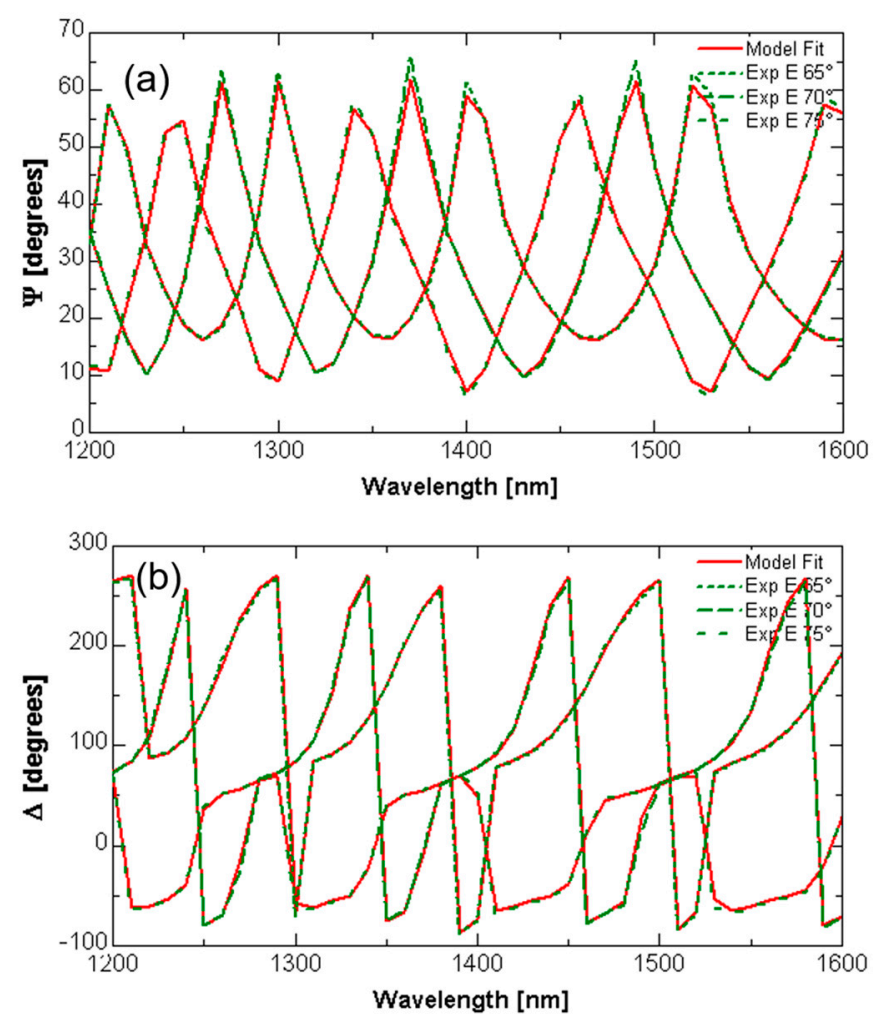

Figure 5. Ellipsometric (a) psi $(\psi)$ and $(\mathbf{b})$ del $(\Delta)$ data acquired at three angles of measurements $\left(65^{\circ}, 70^{\circ}\right.$, and $\left.75^{\circ}\right)$ and their respective model curves (solid red lines) calculated with a four-layer film/substrate optical stack.

The optical constants (n, k) of the deposited SiOC film are shown in Figure 6. The extinction coefficient $\mathrm{k}$ is less than $10^{-4}$ (this value being the minimum detectable value for the ellipsometer) in agreement with previous observations [14] and is not visible in Figure 6. The ordinary axis refractive index $\mathrm{n}_{\mathrm{o}}$ is determined to be 1.554 while it is 1.578 for extra-ordinary axis $\mathrm{n}_{\mathrm{e}}$ at the wavelength $\lambda=1550 \mathrm{~nm}$. The values of dispersion constants A and B of Cauchy model (Equation (2)) are 1.5528 and $4.0353 \times 10^{-3}$, respectively.

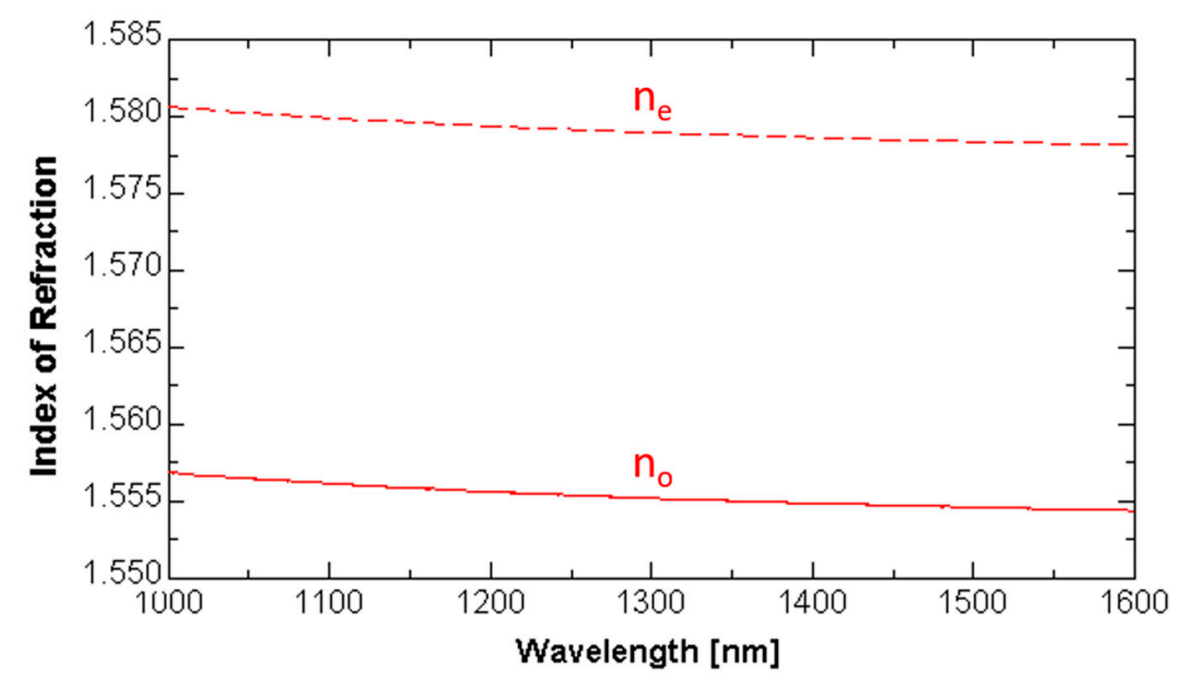

Figure 6. Ordinary $\left(n_{0}\right)$ and extra-ordinary $\left(n_{e}\right)$ refractive index curves of the deposited SiOC film extracted from measured ellipsometric data. 


\subsection{SiOC Optical Waveguides}

The channel waveguides are fabricated in silicon oxycarbide (SiOC) using UV photolithography and reactive ion etching (RIE) techniques as described in Section 2.2. The channel waveguides are realized because they provide high confinement of electromagnetic light wave in two dimensions. The light confinement in the SiOC core region can be enhanced by increasing waveguide core dimensions (width and height).

After each step during the whole process, the optical chips were analyzed under optical microscope to check for the quality, defects, and residual of photoresist. The photomask used to define waveguide patterns with photolithography has a set of hundreds of waveguides with different widths ranging from 1.8 to $4.5 \mu \mathrm{m}$. Figure 7a shows micrograph of the SiOC waveguides (black strips) captured with optical microscope at the end of the waveguide fabrication process (after photoresist lift-off, before upper cladding deposition). The width of the waveguides increases from top to bottom (arrow direction). The waveguides definition is clear and free from defects. High resolution SEM image of one of the SiOC channel waveguides with refractive index $n_{e}=1.578$ and dimensions of $4400 \mathrm{~nm}$ by $400 \mathrm{~nm}$ is shown in Figure $7 \mathrm{~b}$ before the deposition of the silica upper cladding. The SiOC waveguides were buried under $8 \mu \mathrm{m}$ thick upper $\mathrm{SiO}_{2}$ cladding $(\mathrm{n}=1.444)$ and provide an index contrast $\Delta \mathrm{n}$ of $8.2 \%$. The index contrast between core and clad is calculated using the relation [21],

$$
\Delta \mathrm{n}=\frac{\left(\mathrm{n}_{\text {core }}^{2}-\mathrm{n}_{\text {clad }}^{2}\right)}{2 \mathrm{n}_{\text {core }}^{2}}
$$
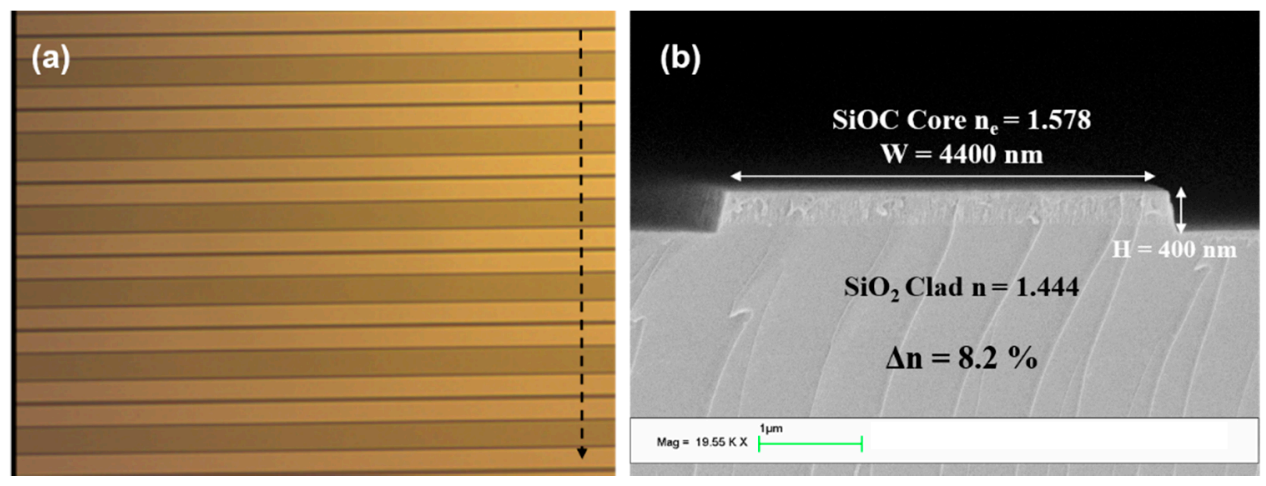

Figure 7. (a) Optical micrograph of straight SiOC waveguides with different widths (increasing from top to bottom with arrow) captured with optical microscope after photoresist lift-off, (b) high resolution SEM image of SiOC channel waveguide with dimensions (width $\mathrm{W}=4400 \mathrm{~nm}$ by height $\mathrm{H}=400 \mathrm{~nm}$ ).

Based on the defined geometry of SiOC waveguides, electromagnetic simulations were performed to understand the mode of operation (single- or multi-mode) of the waveguides and compute the effective refractive index $\mathrm{n}_{\text {eff }}$ values of the propagating TE and TM modes. Figure 8a shows the fundamental TE ad TM modes of the SiOC waveguide with dimensions $4400 \times 400 \mathrm{~nm}^{2}$ (see Figure $7 \mathrm{~b}$ ) and their calculated $\mathrm{n}_{\mathrm{eff}}$ values are 1.458 and 1.4602 at $\lambda=1550 \mathrm{~nm}$. The confinement factor of the SiOC waveguides for the fundamental TE and TM modes is around $30 \%$. In Figure $8 \mathrm{~b}, \mathrm{n}_{\text {eff }}$ of TE and TM modes of the SiOC waveguide are computed as a function of width $(2-4.4 \mu \mathrm{m})$. The SiOC waveguides are single mode as no higher order TE or TM modes appear when the width is varied from 2 to $4.4 \mu \mathrm{m}$. The $\mathrm{n}_{\text {eff }}$ of fundamental TM modes is larger than TE modes due to the anisotropy exhibited by columnar structure in SiOC film that is on the order of $10^{-2}$. 
(a)
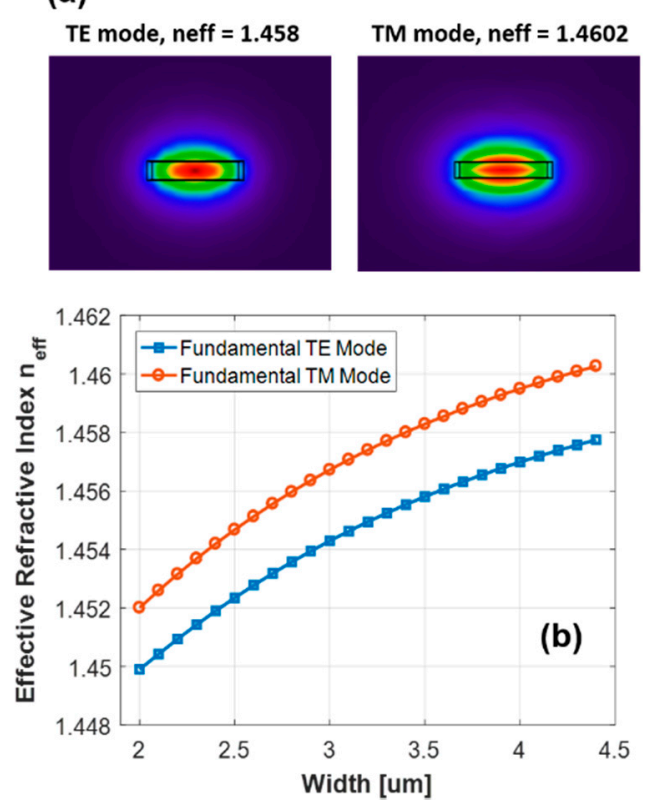

Figure 8. (a) Fundamental TE and TM modes of the SiOC waveguide $\left(\mathrm{W}=4400 \times \mathrm{H}=400 \mathrm{~nm}^{2}\right)$ and their respective effective indices $n_{\text {eff }}$ computed with FEM based software, (b) Plot of effective refractive index $\mathrm{n}_{\mathrm{eff}}$ of SiOC waveguide modes as a function of increasing width $(2-4.4 \mu \mathrm{m})$, only fundamental TE and TM modes exist at $\lambda=1550 \mathrm{~nm}$.

Figure 9 shows the experimental analyses of losses of single mode SiOC channel waveguides for different widths $(2-4.4 \mu \mathrm{m})$ by using a cut-back technique. This technique enables to identify both the propagation losses of the waveguide and the coupling losses due to mismatch between fiber and waveguide modes overlap. As shown in Figure 9a, the propagation losses of SiOC optical waveguides having dimensions of $\mathrm{W}=4400 \mathrm{~nm}$ by $\mathrm{H}=400 \mathrm{~nm}$ are estimated to be $4 \pm 0.5 \mathrm{~dB} / \mathrm{cm}$ from the data slope versus waveguide length. The excited TE and TM polarizations (shown with black right and red left triangles, respectively) exhibit similar propagation losses. The coupling losses due to the mismatch between fiber and waveguide modes are estimated around $1.5 \mathrm{~dB} / \mathrm{facet}$, and are in good agreement with numerical simulations, which predict $80 \%$ coupling efficiency. In general, the coupling efficiency varies between fiber and waveguide modes with waveguide core size. The difference in coupling efficiency among SiOC waveguides with different core widths (between $2 \mu \mathrm{m}$ and $4.4 \mu \mathrm{m}$ ) is $5 \%$ for both polarizations as evaluated from electromagnetic simulations. A difference of $5 \%$ means an additional loss of $0.2 \mathrm{~dB}$ that is within measurement error of our setup and has no appreciable effect on the assessment of waveguides losses. Furthermore, the coupling efficiency for TE and TM polarizations differing by $1 \%$ for respective waveguide width is negligible. The similar coupling efficiency is due to the anisotropy of the material that brings the $\mathrm{n}_{\mathrm{eff}}$ of TE and TM modes closer to each other.

Figure $9 \mathrm{~b}$ shows propagation loss as a function of core width. The average losses for both TE and TM polarizations are similar while there is a small increase in the propagation losses from wider $(4.4 \mu \mathrm{m})$ to narrower $(2 \mu \mathrm{m})$ waveguides. The confinement factor is calculated to decrease by about $8 \%$ from wider to narrower core waveguides for both TE and TM polarizations is a small number and has no impact on losses. The increase in propagation loss in narrower core waveguides can hence be attributed to the sidewall roughness that contribute to scattering of light. Moreover, from ellipsometric measurements the extinction coefficient $\mathrm{k}$ is undetectable which implies that SiOC material losses are low. Therefore, the origin of the waveguide losses is expected from the surface roughness and columnar structure of the sputtered SiOC film. To reduce the waveguide losses, the sputter process may further be optimized to obtain more compact SiOC films, annealing may be considered to smoothen the film structure or CVD process [22]. 
The results on SiOC waveguides indicate the significance of this material for guided wave applications. SiOC optical waveguides with higher refractive index $(n>1.578)$ are under development and will be demonstrated in a future study.
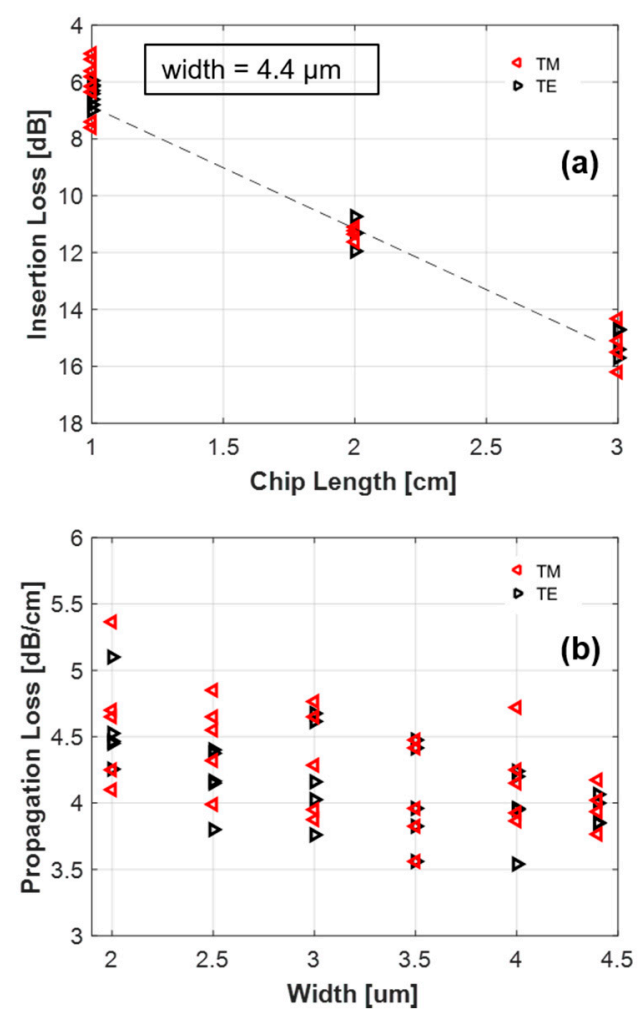

Figure 9. (a) Insertion loss plot of the SiOC waveguides with different chip lengths $(1-3 \mathrm{~cm})$, (b) SiOC waveguides propagation losses as a function of width $(2-4.4 \mu \mathrm{m})$ for both TE and TM polarizations.

\section{Conclusions}

We have demonstrated for the first time the possibility of using silicon oxycarbide ( $\mathrm{SiOC}$ ) as a core material for guided wave applications. Detailed characterization on morphology, topography, and optical properties of sputtered SiOC film are presented. The channel waveguides are fabricated using UV photolithography and reactive ion etching processes. SiOC optical waveguides with core refractive index $\mathrm{n}_{\mathrm{e}}=1.578$ and $\mathrm{SiO}_{2}$ cladding provides a much higher index contrast (of about $8.2 \%$ ) with respect to a conventional glass photonic platform. Moreover, SiOC offers the possibility of tuning the refractive index across a wide range, potentially from 1.45 to $\sim 3.0$, which means that index contrast as high as $38 \%$ can be achieved. Systematic investigation of SiOC waveguides with different widths $(2-4.4 \mu \mathrm{m})$ and lengths $(1-3 \mathrm{~cm})$ is reported. The propagation losses of $4 \pm 0.5 \mathrm{~dB} / \mathrm{cm}$ for both TE and TM polarizations are presented as estimated from cut-back technique. Results indicate the potential of $\mathrm{SiOC}$ as a promising platform for integrated optics.

In order to reduce the losses of SiOC waveguides, the sputter process may further be optimized to achieve uniform films. Losses are perceived to originate mainly due to scattering from surface roughness and columnar structure present in the films. The annealing step may also be considered to smoothen the film structure. The study provides proof of concept for the SiOC photonic platform which may further be developed with CVD.

Higher index contrast waveguides and advanced optical devices in silicon oxycarbide are under development and will be demonstrated in future work. 
Acknowledgments: Faisal Ahmed Memon acknowledges the financial support in his PhD from European Union under Erasmus Mundus 'LEADERS' project. This work was partially performed at Polifab, the micro- and nanofabrication facility of Politecnico di Milano (www.polifab.polimi.it) and the authors thank Claudio Somaschini for the valuable technical support.

Author Contributions: Faisal Ahmed Memon designed and performed the experiments for fabrication and characterizations of silicon oxycarbide thin films and optical waveguides. Faisal Ahmed Memon simulated the optical waveguides in COMSOL educational licensed (PoliMI) version (5.2) software using Wave Optics module based on finite element method (FEM). Francesco Morichetti contributed in measuring the propagation losses of the SiOC waveguides. The results were discussed by Faisal Ahmed Memon, Francesco Morichetti, and Andrea Melloni. The manuscript was written by Faisal Ahmed Memon and edited by Francesco Morichetti and Andrea Melloni.

Conflicts of Interest: The authors declare no conflict of interest.

\section{References}

1. Ng, D.K.; Wang, Q.; Wang, T.; Ng, S.K.; Toh, Y.T.; Lim, K.P.; Yang, Y.; Tan, D.T. Exploring High Refractive Index Silicon-Rich Nitride Films by Low-Temperature Inductively Coupled Plasma Chemical Vapor Deposition and Applications for Integrated Waveguides. ACS App. Mater. Interfaces 2015, 7, 21884-21889. [CrossRef] [PubMed]

2. Wörhoff, K.; Driessen, A.; Lambeck, P.V.; Hilderink, L.T.H.; Linders, P.W.C.; Popma, T.J.A. Plasma enhanced chemical vapor deposition silicon oxynitride optimized for application in integrated optics. Sens. Actuators A Phys. 1999, 74, 9-12. [CrossRef]

3. Pradeep, V.S.; Graczyk-Zajac, M.; Riedel, R.; Soraru, G.D. New Insights in to the Lithium Storage Mechanism in Polymer Derived SiOC Anode Materials. Electrochim. Acta 2014, 119, 78-85. [CrossRef]

4. Peng, Y.; Zhou, J.; Zheng, X.; Zhao, B.; Tan, X. Structure and photoluminescence properties of silicon oxycarbide thin films deposited by the rf reactive sputtering. Int. J. Mod. Phys. B 2011, 25, 2983. [CrossRef]

5. Ding, Y.; Shirai, H.; He, D. White light emission and electrical properties of silicon oxycarbide-based metal-oxide-semiconductor diode. Thin Solid Films 2011, 519, 2513-2515. [CrossRef]

6. Kim, H.J.; Shao, Q.; Kim, Y.-H. Characterization of low-dielectric-constant SiOC thin films deposited by PECVD for interlayer dielectrics of multilevel interconnection. Surf. Coat. Technol. 2003, 171, $39-45$. [CrossRef]

7. Wang, M.R.; Rusli; Xie, J.L.; Babu, N.; Li, C.Y.; Rakesh, K. Study of oxygen influences on carbon doped silicon oxide low $\mathrm{k}$ thin films deposited by plasma enhanced chemical vapor deposition. J. Appl. Phys. 2004, 96, 829-834. [CrossRef]

8. Ryan, J.V.; Pantano, C.G. Synthesis and characterization of inorganic silicon oxycarbide glass thin films by reactive rf-magnetron sputtering. J. Vac. Sci. Technol. A 2007, 25, 153-159. [CrossRef]

9. Gallis, S.; Nikas, V.; Huang, M.; Eisenbraun, E.; Kaloyeros, A.E. Comparative study of the effects of thermal treatment on the optical properties of hydrogenated amorphous silicon-oxycarbide. J. Appl. Phys. 2007, 102, 24302. [CrossRef]

10. Pantano, C.G.; Singh, A.K.; Zhang, H. Silicon Oxycarbide Glasses. J. Sol-Gel. Sci. Technol. 1999, 14, 7-25. [CrossRef]

11. Renlund, G.; Prochazka, S.; Doremus, R. Silicon oxycarbide glasses: Part I. Preparation and chemistry. J. Mat. Res. 1991, 6, 2716-2722. [CrossRef]

12. Renlund, G.; Prochazka, S.; Doremus, R. Silicon oxycarbide glasses: Part II. Structure and properties. J. Mater. Res. 1991, 6, 2723-2734. [CrossRef]

13. Sorarù, G.D.; D’Andrea, G.; Glisenti, A. XPS characterization of gel-derived silicon oxycarbide glasses. Mater. Lett. 1996, 27, 1-5. [CrossRef]

14. Memon, F.A.; Morichetti, F.; Abro, M.I.; Iseni, G.; Somaschini, C.; Aftab, U.; Melloni, A. Synthesis, Characterization and Optical Constants of Silicon Oxycarbide. EPJ Web Conf. 2017, 139. [CrossRef]

15. Fujiwara, H. Spectroscopic Ellipsometry: Principles and Applications; Wiley: West Sussex, UK, 2007.

16. Bruggeman, D.A.G. Berechnung verschiedener physikalischer Konstanten von heterogenen Substanzen I. Dielektrizitätskonstanten und Leitfähigkeiten der Mischkörper aus isotropen Substanzen. Ann. Phys. 1935, 24, 636-679. [CrossRef] 
17. J.A. Woollam Co., Inc. Guide to Using WVASE ${ }^{\circledR}$ Spectroscopic Ellipsometry Data Acquisition and Analysis Software; J.A. Woollam Co., Inc.: Lincoln, NE, USA, 1994-2012.

18. Valyukh, I.; Green, S.; Arwin, H.; Niklasson, G.A.; Wäckelgård, E.; Granqvist, C.G. Spectroscopic ellipsometry characterization of electrochromic tungsten oxide and nickel oxide thin films made by sputter deposition. Sol. Energy Mater. Sol. Cells 2010, 94, 724-732. [CrossRef]

19. Liu, T.; Henderson, C.L.; Samuels, R. Quantitative characterization of the optical properties of absorbing polymer films: Comparative investigation of the internal reflection intensity analysis method. J. Polym. Sci. B Polym. Phys. 2003, 41, 842-855. [CrossRef]

20. Prest, W.M., Jr.; Luca, D.J. The origin of the optical anisotropy of solvent cast polymeric films. J. Appl. Phys. 1979, 50, 6067. [CrossRef]

21. Senior, J.M. Optical Fiber Communications: Principles and Practice, 3rd ed.; Pearson Education Limited: Edinburgh, UK, 2009; pp. 19-20.

22. Mandracci, P.; Frascella, F.; Rizzo, R.; Virga, A.; Rivolo, P.; Descrovi, E.; Giorgis, F. Optical and structural properties of amorphous silicon-nitrides and silicon-oxycarbides: Application of multilayer structures for the coupling of Bloch Surface Waves. J. Non-Cryst. Solids 2016, 453, 113-117. [CrossRef]

(C) 2017 by the authors. Licensee MDPI, Basel, Switzerland. This article is an open access article distributed under the terms and conditions of the Creative Commons Attribution (CC BY) license (http:/ / creativecommons.org/licenses/by/4.0/). 